\title{
Giant cell tumor of dorsal vertebrae-a rare case report with review of literature
}

\begin{abstract}
A 32-year-old well-built male presented to the Orthopaedics clinic with major complaints of low backache with both lower limbs weakness and disturbed bladder/ bowel habits. An osteolytic lesion was seen at tenth thoracic vertebra with anterior compression on the spinal cord on radio-imaging. Giant cell tumor (GCT) of dorsal vertebral body is an uncommon entity and total en bloc excision is difficult. Complete intralesional tumor excision with reconstruction was performed. Histopathological examination was suggestive of GCT. Our patient is doing well after one year of follow-up, without any signs of recurrence of the tumor and is ambulant with support. We discuss in brief about incidence, presentation and various treatment modalities available for spinal GCT.
\end{abstract}

Volume 2 Issue 2 - 2017

Kafil Akhtar, Lateef Z Jilani, Mazhar Abbas, Rana K Sherwani

Department of Pathology and Orthopaedics Surgery, Aligarh Muslim University, India

Correspondence: Kafil Akhtar, Department of Pathology, Jawaharlal Nehru Medical College, Aligarh Muslim University, Aligarh (UP), India; Email drkafilakhtar@gmail.com

Received: November 04, 2016 | Published: March 22, 2017

Keywords: dorsal vertebral body, giant cell tumor, histopathology

\section{Introduction}

Giant cell tumor (GCT) is locally malignant tumor, which commonly involves the ends of long bone. It is locally aggressive, and tumor recurrence is frequently seen after intralesional or incomplete excision. ${ }^{1}$ Most common site for vertebral GCT is the sacrum. They are distinctly rare above the sacrum and present with destruction of vertebral body and neural arch. The treatment of choice is total spondylectomy with appropriate reconstruction for preservation of spinal integrity. Adjuvant therapy in the form of radiation, can be given in cases of subtotal resection. ${ }^{2} \mathrm{GCT}$ are known to be aggressive in behaviour, carry some malignant potential with a high incidence of local recurrence. They affect the spinal axis in $8 \%$ to $11 \%$ of cases and occur in the third and fourth decades of life, with a female preponderance. ${ }^{2,3}$ Long bones are the most common site of GCT of bone and spine is the fourth leading location of GCT, with sacrum the prime foci of insult. ${ }^{1}$ Only $1 \%$ to $2 \%$ of GCTs have been reported in the thoracic spine..$^{2-4}$ GCTs of the spine sometimes extend into the paraspinal soft tissue. ${ }^{1}$

\section{Case summary}

A 32-year-old male presented to the Orthopaedics clinic with chief complaints of low backache with both lower limbs weakness and disturbed bladder/bowel habits for 4 months duration. General examination was unremarkable. On local examination, he had tenderness over lower dorsal region and power of grade 2-3 in both lower limbs. An anterior-posterior and lateral X-ray showed purely osteolytic bone deficit with unclear boundaries associated with collapse of the posterolateral tenth lumbar vertebra (Figure 1). Magnetic resonance imaging (MRI) was suggestive of osteolytic lesion involving tenth dorsal vertebral body and left neural arch with extension into posterior mediastinal space (Figure 2). Fine needle aspiration biopsy from the lesion showed multiple osteoclastic giant cells, spindle cells and foci of haemorrhage, suggestive of giant cell lesion of the bone (Figure 3). Left anterolateral approach with excision biopsy of the soft moderately vascular osteolytic tumor was performed (Figure 4). Histopathological examination showed multiple haphazardly arranged osteoclasts like multinucleated giant cells and atypical plump to spindle shaped stromal cells, suggestive of GCT. Patient improved clinically with power of 4-5 in both lower limbs after surgery (Figure 5). Radiation therapy was not given in the present case due to fear of osteoradionecrosis and myelitis. Our patient is asymptomatic after 1 year of follow up, without any evidence of local and distant tumor recurrence.

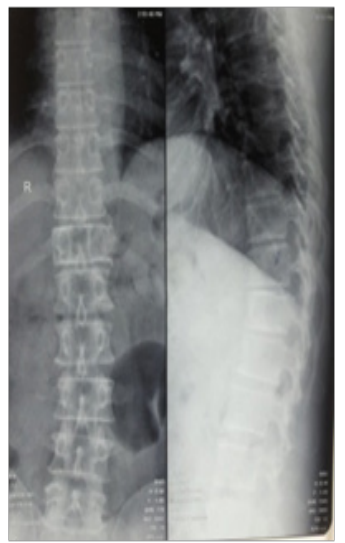

Figure I An anterior-posterior and lateral X-ray showed purely osteolytic bone deficit with unclear boundaries associated with collapse of the posterolateral tenth lumbar vertebra.

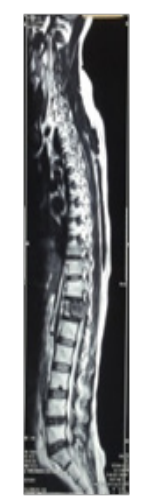

Figure 2 Magnetic resonance imaging (MRI) showed an osteolytic lesion involving tenth dorsal vertebral body and left neural arch with extension into posterior mediastinal space. 


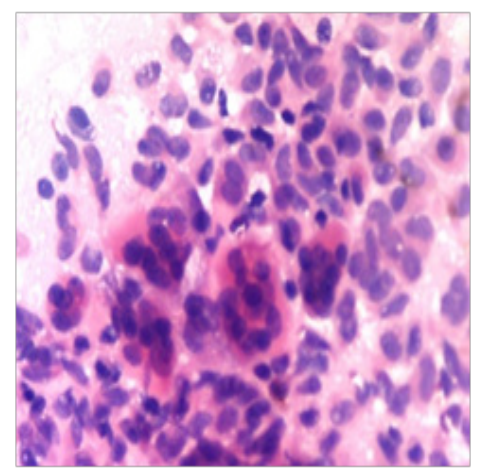

Figure 3 Fine needle aspiration biopsy from the lesion showed multiple osteoclastic giant cells, spindle cells and foci of haemorrhage, suggestive of giant cell lesion of the bone, H\&E x 40X.

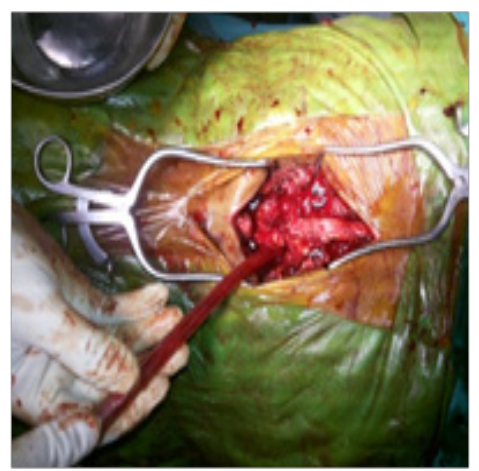

Figure $4 \mathrm{~A}$ per-operative left anterolateral approach with excision biopsy of the soft moderately vascular osteolytic tumor was performed.

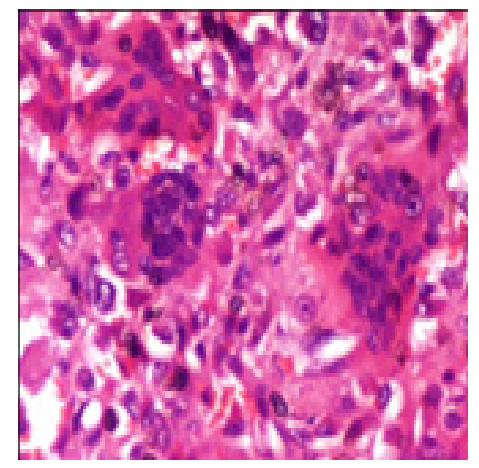

Figure 5 Histopathological examination showed multiple haphazardly arranged osteoclastic giant cells and atypical plump to spindle shaped stromal cells, suggestive of Giant cell tumor of the bone, H\&E x 40X.

\section{Discussion}

The incidence rate of GCT of bone is $5.0 \%$ amongst all primary bone tumors and only $1-1.5 \%$ GCTs occur in the mobile spinal segment. ${ }^{1}$ Roughly, equal incidence is seen in all three mobile spinal segments above the sacrum. They affect the spinal axis in $8 \%$ to $11 \%$ of cases and occur in the third and fourth decades of life, with a female preponderance. ${ }^{2,3}$ Common symptoms include, back pain, neurological deficit due to compression of spinal cord, bladder and bowel dysfunction, and structural deformity of the spine. GCT usually arise in the epiphysis of the long bones (75\%) and spinal GCT is reported in only two to three percent cases. ${ }^{3}$ Spinal GCT mostly occurs in the thoracic region followed by lumbar and cervical vertebrae. ${ }^{4,5}$ The most common presenting features of spinal GCT are localized pain and progressive paraparesis. The disease has a female predominance, in the age group of 20-40 years. ${ }^{3}$

Giant cell tumors are also known as osteoclastomas as these are considered to arise from the osteoclast. The exact cell of origin, however, is unclear. ${ }^{4,5}$ The histologic appearance of GCT is a uniform distribution of multinucleated giant cells against a background of round to spindle cells shaped atypical mononuclear stromal cells. Haphazardly arranged multinucleated giant cells but benign looking oval to plump stromal cells are also seen in few benign bone lesions like Osteochondromas, osteoblastoma, chondroblastoma, chondromyxoid fibroma, brown tumor of hyperparathyroidism, eosinophilic granuloma, fibrous dysplasia and aneurysmal bone cysts. ${ }^{3,4}$ Spinal GCT on radiographic image is seen as round or oval mass with shell-like calcification devoid of mineralized matrix. ${ }^{5}$ As opposed to other tumors, they involve the vertebral body and softtissue involvement may be present. Histological confirmation of the diagnosis requires a surgical biopsy or a CT scan guided puncture biopsy, whose reliability is $65 \% 0^{2,5}$ We performed a surgical biopsy along with decompressive laminectomy. A CT scan guided puncture biopsy is safe, and can be performed to confirm tumor recurrence. ${ }^{5,6}$ In most cases, the histological examination confirms the diagnosis of GCT and excludes the main differential diagnoses, in particular aneurysmal cyst. ${ }^{3,4}$

The different recommended treatment protocols for spinal GCTs are surgery, radiotherapy, cryosurgery, cementation, and chemical adjuvant therapy. Total en bloc surgical debridement is the treatment of choice in long bones as well as spine but is not always feasible in the spine due to the unacceptable risk of permanent neurological deficit. ${ }^{3}$ But Christopher and Edward have stated that Giant cell tumors of the spine and sacrum should be managed with en bloc resections whenever possible as this provides the greatest chance for cure. ${ }^{7}$ Simple curettage can lead to local recurrence in $24-40 \%$ cases, which can be minimized by adjuvant treatment of cement implantation and chemical therapy. ${ }^{8}$ Due to the risk of myelitis and bone graft complications, adjuvant radiotherapy should be reserved for incomplete tumor excision and local recurrence. ${ }^{9110}$ Lungs are the common sites of metastases from GCTs, in about $2.0-9.0 \%$ of cases. ${ }^{9,10}$ Close follow-up is required to detect recurrence of the tumor. Donthineni et al noted higher incidence of lung metastases from spinal GCT as compared to long bones. ${ }^{6}$ Metastasectomy of the lung nodules can be considered in view of prolonged survival. Plain radiograph of local site and chest are simple tools to look for any recurrent lesion. Periodic computed tomography and MRI are excellent tools to clearly identify the recurrent lesion and plan necessary treatment. ${ }^{11,12}$ In the present case, total tumor excision was achieved by intralesional approach and solid reconstruction was achieved, using pedicular screw and rod fixation.

\section{Acknowledgements}

None.

\section{Conflict of interest}

The author declares no conflict of interest.

\section{References}

1. Mendenhall WM, Zlotecki RA, Scarborough MT, Giant cell tumor of bone. Am J Clin Oncol. 2006;29(1):96-99.

2. Campanacci M, Baldini N, Boriani S, et al. Giant-cell tumor of bone. $J$ Bone Joint Surg Am. 1987;69(1):106-114. 
3. Boriani S, Weinstein JN, Biagini R. Primary bone tumors of the spine. Terminology and surgical staging. Spine. 1997;22(9):1036-1044.

4. Sobti S, John PS, Kumar A, et al. Giant cell tumor of dorsal spine. $J$ Spine Surg. 2016;3(3):112-113.

5. A Soltani, S Adel, M Mashari, M Panahbekhoda, et al. Giant-Cell Tumor of Third Lumbar Vertebrae: A Case Report. The Internet Journal of Spine Surgery. 2009;5(2):22-24.

6. Donthineni R, Boriani L, Ofluoglu O, et al. Metastatic behaviour of giant cell tumour of the spine. Int Orthop. 2009;33(2):497-501.

7. Christopher M, Edward M. Giant cell tumor of the sacrum and spine: series of 23 cases and a review of the literature. Iowa Orthop J. 2010;30:69-71
8. Redhu R, Poonia R. Giant cell tumor of dorsal vertebral body. J Craniovert Jun Spine. 2012;3(2):67-69

9. Schutte HE, Taconis WK. Giant cell tumor in children and adolescents. Skeletal Radiol. 1993;22(3):173-176

10. Fidler MW. Surgical treatment of giant cell tumours of the thoracic and lumbar spine: Report of nine patients. Eur Spine J. 2001;10(1):69-77.

11. Murphey MD, Andrews CL, Flemming DJ, et al. From the archives of the AFIP. Primary tumors of the spine. Radiologic pathologic correlation. Radiographics. 1996;16(5):1131-1158.

12. Jong WK, Hye WC, Eun YC, et al. MRI Findings of Giant Cell Tumors of the Spine. Am J Roentgenol. 2007;189(1):246-250. 